\title{
Increasing HER2 $\alpha 2,6$ sialylation facilitates gastric cancer progression and resistance via the Akt and ERK pathways
}

\author{
NAIHUA LIU ${ }^{1 *}$, MENGLU ZHU $^{2 *}$, YINGJIE LINHAI $^{3}$, YUWEI SONG $^{4}$, XIUJUAN GUI $^{4}$, GUOQIANG TAN $^{4}$, \\ JINYUAN LI ${ }^{4}$, YAN LIU ${ }^{4}$, ZHENDONG DENG ${ }^{1}$, XIAOTING CHEN ${ }^{1}$, JING WANG $^{2}$, LILI JIA ${ }^{2}$, \\ XIUZHEN HE ${ }^{2}$, XIAOYU WANG ${ }^{4}$ and SHAOQIANG LIN ${ }^{1,2,4}$ \\ ${ }^{1}$ Clinical Department of Guangdong Metabolic Disease Research Center of Integrated Chinese and Western Medicine, \\ The First Affiliated Hospital of Guangdong Pharmaceutical University, Guangzhou, Guangdong 510080; \\ ${ }^{2}$ School of Pharmaceutical Sciences of Wenzhou Medical University, Wenzhou, Zhejiang 325035; \\ ${ }^{3}$ Department of Orthopedics, Third Affiliated Hospital of Southern Medical University, Guangzhou, Guangdong 510630; \\ ${ }^{4}$ The First Affiliated Hospital of Jinan University, Guangzhou, Guangdong 510632, P.R. China
}

Received February 14, 2018; Accepted August 3, 2018

DOI: $10.3892 / o r .2018 .6680$

\begin{abstract}
Upregulated $\beta$-galactoside $\alpha 2,6$-sialyltransferase I (ST6Gal-I) expression reportedly occurs in many cancers and is correlated with metastasis and poor prognosis. However, the mechanisms by which ST6Gal-I facilitates gastric cancer progression remain poorly understood. Trastuzumab is exclusively used in human epidermal growth factor receptor 2 (HER 2) $^{+}$gastric cancers; however, most advanced HER2 ${ }^{+}$ gastric cancers develop trastuzumab resistance. Herein, we identified HER2 as an ST6Gal-I substrate and showed that HER2 $\alpha 2,6$ sialylation confers protection against trastuzumab-mediated apoptosis. SGC7901 cancer cell models in which ST6Gal-I was overexpressed or knocked down were constructed, revealing that ST6Gal-I overexpression induced high HER2 sialylation levels and increased cell viability and invasion compared to those in the vector cell line under serum starvation; ST6Gal-I knockdown had the opposite effects.
\end{abstract}

Correspondence to: Dr Shaoqiang Lin, Clinical Department of Guangdong Metabolic Disease Research Center of Integrated Chinese and Western Medicine, The First Affiliated Hospital of Guangdong Pharmaceutical University, 19 Nonglinxia Road, Guangzhou, Guangdong 510080, P.R. China

E-mail: sqlin123@163.com

Dr Xiaoyu Wang, The First Affiliated Hospital of Jinan University, 613 West Huangpu Avenue, Guangzhou, Guangdong 510632, P.R. China

E-mail: twxy@jnu.edu.cn

*Contributed equally

Abbreviations: ST6Gal-I, $\beta$-galactoside $\alpha 2,6$-sialyltransferase; HER2, human epidermal growth factor receptor 2

Key words: gastric cancers, ST6Gal-I, HER2, cancer progression, drug resistance
ST6Gal-I overexpression also potentiated cell cycle arrest in the $\mathrm{G} 2 / \mathrm{S}$ phase to reduce drug sensitivity. In addition, FACS analysis revealed that high ST6Gal-I levels increased resistance to trastuzumab-induced apoptosis, accompanied by decreased caspase-3 levels. However, the ST6Gal-I knockdown cell line revealed increased caspase-3 levels and evident apoptosis compared with those in the vector cell line. Although ST6Gal-I overexpression increased HER2 sialylation, corresponding to decreased HER2 phosphorylation, high $\alpha 2,6$-sialylation enhanced Akt and ERK phosphorylation levels compared to those in the vector cell line; ST6Gal-I knockdown had the opposite effects. Collectively, these results implicated a functional role of ST6Gal-I in promoting tumor cell progression and trastuzumab resistance.

\section{Introduction}

Gastric cancer (GC), one of the most common cancer types diagnosed in humans worldwide, is associated with poor prognosis and metastasis $(1,2)$. While significant progress has been achieved, treatments for GC are not yet satisfactory. The average 5-year survival rate of advanced-stage patients is only $\sim 5-20 \%$ (3), which is accompanied by tumor metastasis and drug resistance.

Human epidermal growth factor receptor 2 (HER2) has transmembrane tyrosine kinase activity that promotes cell proliferation and migration (4). HER2 is a proto-oncogene present on chromosome 17, and overexpression or mutation of HER 2 or abnormal production of HER 2 ligands potentially leads to tumorigenesis in many tissues (5). These factors even play vital roles in the development and drug resistance of GC (6). Approximately 7-34\% of GCs are characterized by poor prognosis associated with amplification of the HER2 gene $(7,8)$. Chemotherapy in combination with trastuzumab, a humanized monoclonal antibody against HER2, carries a significant survival advantage; however, $12 \%$ of all HER2-positive GC cases still exhibit cancer progression, cancer recurrence or subsequent drug resistance (9). Thus, 
we herein aimed to further study the molecular mechanisms underlying HER2 in GC to develop more effective treatment for patients.

Abnormal cellular glycosylation plays a key role in cancer progression and malignancy (10-12). Glycosylation is regulated by various glycosyltransferases, such as fucosyl-, sialyl- and galactosyltransferases, which catalyze the transfer of monosaccharide residues from nucleotide sugar donors to specific acceptor substrates, forming glycosidic bonds. In addition, sialyltransferases are the key enzymes that transfer sialic acid from CMP-NeuAc2 to glycoproteins or glycolipids in the biosynthesis of sialic acid-containing glycoproteins and glycolipids, which are altered in carcinoma cells of different origins (13) and specifically correlated with carcinoma differentiation metastatic phenotypes (14). One important glycosyltransferase dysregulated in cancer cells is human $\beta$-galactoside $\alpha 2,6$-sialyltransferase (ST6Gal-I) $(15,16)$, whose activity is low or absent in only normal cells and high in metastatic tumor cells, such as breast, liver and colon cancer cells $(12,17-19)$. ST6Gal-I is hypothesized to improve the progression and metastasis of cancer cells via the sialylation-dependent modulation of cell surface receptors. For example, ST6Gal-I overexpression was revealed to increase the $\alpha 2,6$-sialylation levels on the Fas receptor, certain integrins, and TNFR1 impeded apoptosis and blocked TNF-stimulated cell death (20-22). Furthermore, ST6Gal-I was revealed to induce the sialylation of EGFR, resulting in resistance to EGFR-targeted chemotherapy (23). However, the molecular mechanisms by which ST6Gal-I mediates HER2 in GC cells have not been well-characterized.

In addition, determining whether HER $2 \alpha 2,6$-sialylation plays a vital role in the malignancy of GCs is necessary. To identify whether ST6Gal-I is responsible for HER2 sialylation, we prepared and characterized ST6Gal-I knockdown and ST6Gal-I overexpression SGC7901 gastric carcinoma cells. Given that HER2 activity and downstream signaling are highly correlated with cell proliferation, we investigated the effects of HER2 sialylation on the PI3K/Akt pathway and on trastuzumab sensitivity in SGC7901 cancer cells. Our results revealed that the HER2 sialylation level along with HER2 mutations may be a reliable biomarker for anti-HER2 therapy in GC.

\section{Materials and methods}

Cell culture and transfection. SGC7901 GC cells, purchased from the American Type Culture Collection (ATCC; Manassas, VA, USA), were maintained in RPMI-1640 medium with $10 \%$ FBS and $1 \%$ antibiotic solution containing penicillin/streptomycin (all from Gibco; Thermo Fisher Scientific, Inc., Waltham, MA, USA). All cells were cultured in a humidified incubator at $37^{\circ} \mathrm{C}$ with $5 \% \mathrm{CO}_{2}$. The stable ST6Gal-I overexpression, knockdown and empty vector cell lines were established as previously described (24). In brief, the pcDNA3.1(-)/ST6Gal-I, sh-ST6Gal-I and empty vectors were transfected into SGC7901 cancer cells using Lipofectamine 2000 (Invitrogen; Thermo Fisher Scientific, Inc.). Limiting dilution was applied to obtain sub-cell line clones after $24 \mathrm{~h}$ of transfection. Blasticidin $\mathrm{S} \mathrm{HCl}(1.5 \mu \mathrm{g} / \mathrm{ml})$ was used to select the low-expressing ST6Gal-I clone and G418 $(350 \mu \mathrm{g} / \mathrm{ml})$ was utilized to select the ST6Gal-I overexpression clone. ST6Gal-I overexpression or knockdown was verified by ST6Gal-I mRNA expression and protein expression analyses.

Quantitative real-time PCR. Total RNA was isolated using TRIzol reagent (Invitrogen; Thermo Fisher Scientific, Inc.), and cDNA was synthesized using the PrimerScript ${ }^{\circledR}$ RT Master Mix kit (Takara Bio, Inc., Otsu, Japan) according to the manufacturer's protocol. qRT-PCR was performed on a Real-Time PCR Detection System (Bio-Rad Laboratories, Inc., Hercules, CA, USA). The PCR condition was as following: $95^{\circ} \mathrm{C}$ for $5 \mathrm{~min}$, followed by 40 cycles of $95^{\circ} \mathrm{C}$ for $15 \mathrm{sec}, 50^{\circ} \mathrm{C}$ for $30 \mathrm{sec}$ and $72^{\circ} \mathrm{C}$ for $30 \mathrm{sec}$. The sequences of primers used for the real-time PCR assays were as follows: ST6Gal-I: forward, 5'-CCTCTGGGATGCTTGGTATC-3' and reverse, 5'-GTGCAGGCACTATCGAAGAA-3'; GAPDH: forward, 5'-AGCCTCAAGATCATCAGC-3' and reverse, 5'-GAGTCCTTCCACGATACC-3'.

ST6Gal-I activity assay. We conducted lectin staining to assess ST6Gal-I activity. Briefly, the cells were stained with FITC-conjugated SNA lectin (EY Laboratories, San Mateo, CA, USA), which is specific for 2,6-sialic acids, according to the manufacturer's instructions. Cells were stained for $40 \mathrm{~min}$ at $4^{\circ} \mathrm{C}$ with SNA-FITC at a 1:200 dilution and analyzed by fluorescence-activated cell sorting (FACS; BD Biosciences, Franklin Lakes, NJ, USA). In addition, cells were stained with SNA-FITC at a 1:100 dilution for $4 \mathrm{~h}$ for the cell immunofluorescence assay.

Cell Counting Kit-8 (CCK-8) assay. To assess the proliferation of the transfected cloned cell lines, a CCK-8 detection kit (Dojindo Laboratories, Kumamoto, Japan) was used according to the manufacturer's instructions. Approximately 3,000 cells were seeded onto a 96-well plate in quintuplicate for $6 \mathrm{~h}$, and complete medium was then replaced with RPMI-1640 medium without fetal bovine serum (FBS) for $0,24,48,72,96,120$ or $144 \mathrm{~h}$. Next, $10 \mu \mathrm{l}$ of CCK-8 reagent was added to each well, and the absorbance was measured at $450 \mathrm{~nm}$ using a Multiskan Spectrum spectrophotometer (BioTek Instruments, Inc., Winooski, VT, USA). To assess drug resistance, cells were seeded and incubated with different doses of trastuzumab (Sigma-Aldrich; Merck KGaA, Darmstadt, Germany) for $24 \mathrm{~h}$ and CCK-8 assays were then performed.

Cell cycle and apoptosis analysis by flow cytometry. To assess the cell cycle, ST6Gal-I overexpression or ST6Gal-I knockdown cells were seeded onto 6-well plates and cultured in medium containing no FBS for $24 \mathrm{~h}$. The cells were harvested, fixed with $70 \%$ cold ethanol at $4^{\circ} \mathrm{C}$ for $2 \mathrm{~h}$, washed with ice cold phosphate-buffered saline (PBS) 3 times and incubated with staining solution $(20 \mathrm{mg} / \mathrm{ml}$ propidium iodide and $200 \mu \mathrm{g} / \mathrm{ml}$ DNase-free RNase-A in PBS) at room temperature for $30 \mathrm{~min}$ at $37^{\circ} \mathrm{C}$. The labeled cell cycle populations were quantified by FACSCalibur flow cytometry (BD Biosciences) and cell cycle distributions were analyzed by FlowJo software (Tree Star, Inc., Ashland, OR, USA). In addition to the cell apoptosis assays, cells were incubated with trastuzumab for $24 \mathrm{~h}$, collected for staining, and stained with an FITC-labeled Annexin V and propidium iodide staining kit (BD Biosciences, 
San Jose, CA, USA) for $60 \mathrm{~min}$ at $37^{\circ} \mathrm{C}$. Staining controls were prepared with fixed cells single stained and unstained; a positive apoptotic control was obtained by incubating the cells with $1 \mathrm{mM}$ hydrogen peroxide for $15 \mathrm{~h}$ (Sigma-Aldrich; Merck KGaA). Cell populations stained by the 2 different reagents were recorded by FACS.

Transwell invasion assay. In vitro invasion assays were performed using Transwell-24 plates (Corning Inc., Corning, NY, USA); $\sim 5 \times 10^{4}$ transfected cells in $150 \mu \mathrm{l}$ of RPMI-1640 medium without FBS were added to the upper compartment of the Transwell chamber coated with $40 \mu \mathrm{l}$ of Matrigel (pore size, $8.0 \mu \mathrm{m}$; diameter, $6.5 \mathrm{~mm}$ ), and $350 \mu \mathrm{l}$ of RPMI-1640 medium supplemented with $20 \%$ FBS was added to the lower compartment as a chemoattractant. After incubation for $24 \mathrm{~h}$ at $37^{\circ} \mathrm{C}$ and $5 \% \mathrm{CO}_{2}$, the upper chamber was washed 3 times with PBS, and the cells that did not invade through the pores of the upper surface of the membrane were removed by cotton swabs. The invaded cells were subsequently fixed and stained with $0.1 \%$ crystal violet. After being washed with PBS and dried, the cells were visually counted in 4 randomly selected fields with an inverted microscope (Olympus IX71; Olympus Corp., Tokyo, Japan) at x200 magnification.

Western blot analysis. Cells were incubated with trastuzumab for $24 \mathrm{~h}$ and collected for western blot analysis. The cells were lysed using cell lysis buffer, and protein concentrations were assessed with a BCA assay kit (both from Beyotime Institute of Biotechnology, Shanghai, China). Equal amounts of denatured proteins were separated by $10 \%$ SDS-PAGE and transferred to polyvinylidene fluoride (PVDF) membranes. The membranes were blocked with $5 \%$ non-fat milk and then incubated overnight with primary antibodies directed against ST6Gal-I (dilution at 1:500; cat. no. sc-6263), ERK (dilution at 1:1,000; cat. no. sc-514302), p-ERK (dilution at 1:500; cat. no. sc-81492), AKT (dilution at 1:1000; cat. no. sc-5298), p-AKT (dilution at 1:500; cat. no. sc-135650), HER2 (dilution at 1:500; cat. no. sc-7301), p-HER2 (dilution at 1:500; cat. no. sc-81507), caspase-3 (dilution at 1:400; cat. no. sc-7272; all from Santa Cruz Biotechnology, Inc., Santa Cruz, CA, USA) and GAPDH (dilution at 1:2,000; cat. no. ab9484; Abcam, Cambridge, MA, USA), after washed with TBS-Tween $(0.1 \%)$ three times, all membranes were then incubated with

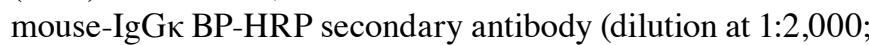
cat. no. sc-516102; Santa Cruz Biotechnology, Inc.) for $1 \mathrm{~h}$ at room temperature. Proteins were visualized using an enhanced chemiluminescence (ECL) kit (Amersham Biosciences, Little Chalfont, UK) according to the manufacturer's instructions. The relative amounts of proteins were determined by densitometry using ImageJ software (National Institutes of Health, Bethesda, MD, USA).

Immunoprecipitation assays. Cells were lysed, and $500 \mu \mathrm{g}$ of lysed protein was incubated overnight at $4^{\circ} \mathrm{C}$ with $50 \mu \mathrm{l}$ of SNA-conjugated agarose (EY Laboratories). $\alpha 2,6$-Sialylated proteins bound to SNA-agarose beads were precipitated by centrifugation and washed extensively with lysis buffer. Sialylated proteins were released from complexes by boiling in SDS-PAGE sample buffer and immunoblotted for HER2 (Santa Cruz Biotechnology, Inc.)
Statistical analysis. Statistical analyses were performed using SPSS 17.0 software (SPSS, Inc., Chicago, IL, USA). The data were presented as the mean value \pm standard deviation (SD). Statistical comparisons between different groups were performed by one-way analysis of variance (ANOVA) with the Least Significant Difference (LSD) post hoc test and statistical comparisons between two groups were analyzed by t-test comparison. Differences were considered statistically significant when $\mathrm{P}$-values were $<0.05$.

\section{Results}

Establishment of ST6Gal-I overexpression and ST6Gal-I knockdown clones of SGC7901 human GC cells. ST6Gal-I and HER2 are biomarkers associated with the malignant progression and poor prognosis of GC (25-27). We confirmed that HER2, p-HER2 and SNA-HER2 were expressed in SGC7901 GC cell lines, MCF7 breast cancer cell lines, and A549 and H460 lung cancer cell lines (Fig. 1A). To evaluate the role of ST6Gal-I-induced sialylation in regulating GC development, we constructed and transfected ST6Gal-I overexpression or ST6Gal-I knockdown plasmids into SGC7901 cells. After limiting dilution and persistent culture, we obtained several different sub-cell line clones with stable expression. The vector 2 cell line was selected as the control group, the X61-4 cell line was selected as the knockdown group, and the PS7 cell line was selected as the overexpression group. ST6Gal-I expression was further confirmed by PCR and western blotting. Endogenous ST6Gal-I gene and protein expression were stably knocked down in the X61-4 cell line, while the PS7 cell line expressed the ST6Gal-I gene and protein at high levels compared to that in the vector cell line (Fig. 1B-E). Collectively, these data strongly demonstrated that ST6Gal-I was stably overexpressed or knocked down in SGC7901 cells.

Overexpression of ST6Gal-I impacts the $\alpha 2,6$-linked sialic acids levels on HER2. To assess the functional consequence of ST6Gal-I upregulation or downregulation in tumor cells, we measured the levels of $\alpha 2,6$-sialylation on the HER 2 receptor. FITC-conjugated SNA lectin was used to recognize $\alpha 2,6$-linked sialic acids using flow cytometry and immunofluorescence microscopy. The FACS results indicated that the SNA fluorescence in PS7 cells was stronger than that in the X61-4 and vector groups (Fig. 2A and B). The immunofluorescence results also revealed that PS7 cells expressed significantly higher levels of $\alpha 2,6$-linked sialic acids than the vector cells, while X61-4 cells expressed lower levels of $\alpha 2,6$-linked sialic acids than the vector control cells (Fig. 2C and D). Moreover, X61-4 cells and PS7 cells were incubated with agarose-conjugated SNA lectin. The $\alpha 2,6$-sialylated proteins bound by SNA-agarose were isolated by SDS-PAGE and immunoblotted for HER2. The SNA-HER2 expression in PS7 cells was much higher than that in the vector group, and SNA-HER2 expression in X61-4 cells was lower than that in the vector group (Fig. 2E). Thus, overexpressing ST6Gal-I hypersialylated HER2, while knocking down ST6Gal-I hyposialylated HER2.

Overexpression of ST6Gal-I impacts the tumor cell cycle and invasion ability. To investigate whether ST6Gal-I protected 


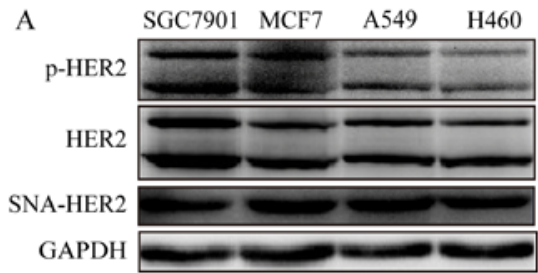

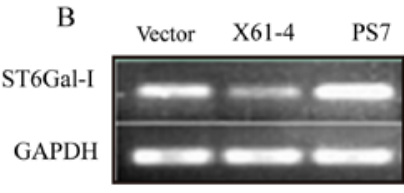

C

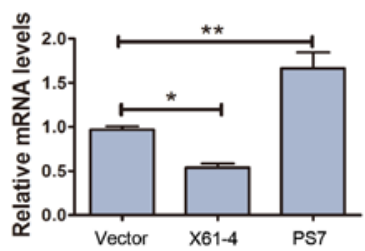

$\mathrm{D}$

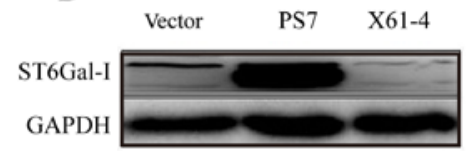

E

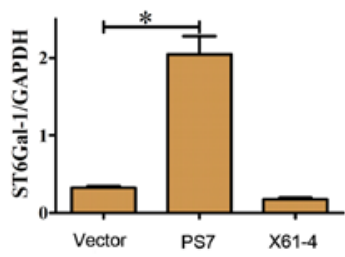

Figure 1. Establishment of ST6Gal-I overexpression and ST6Gal-I knockdown clones of SGC7901 human gastric cancer cells. (A) The expression levels of HER2, p-HER2 and SNA-HER2 in SGC7901, MCF7 and A549 cancer cell lines as determined by western blotting. (B-E) The vector, X61-4 and PS7 cell lines were chosen from stable cell clones of the empty vector group, the ST6Gal-I knockdown group and the ST6Gal-I overexpression group, respectively, and the mRNA and protein levels of ST6Gal-I in the three groups were assessed by PCR and western blotting. ${ }^{*} \mathrm{P}<0.05,{ }^{* * *} \mathrm{P}<0.01$. ST6Gal-I, $\beta$-galactoside $\alpha 2,6$-sialylt ransferase; HER2, human epidermal growth factor receptor 2.

A

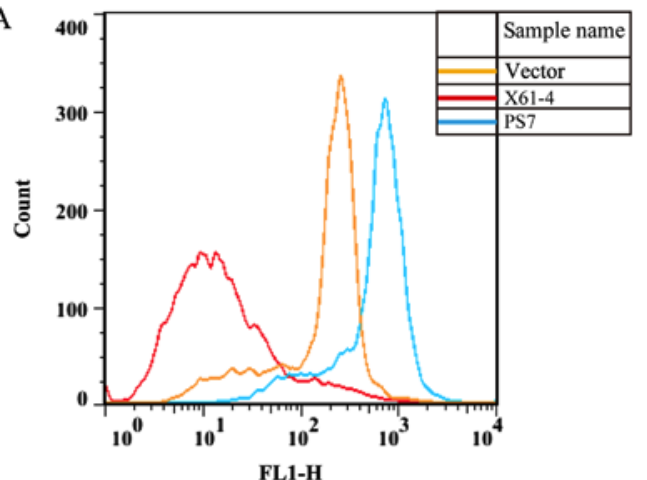

$\mathrm{C}$

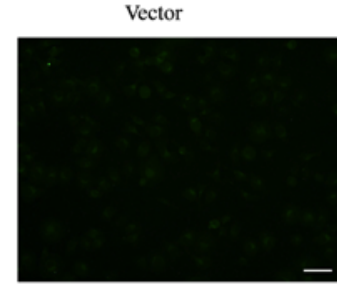

$\mathrm{D}$

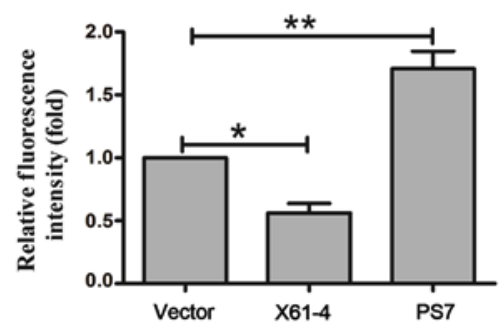

B

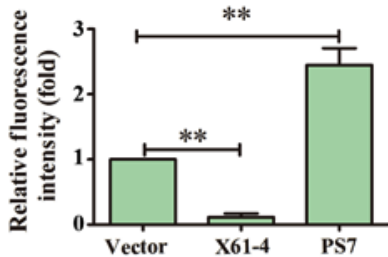

PS7

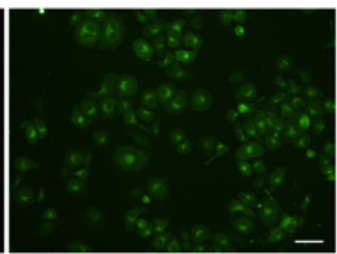

PS7

E Vector
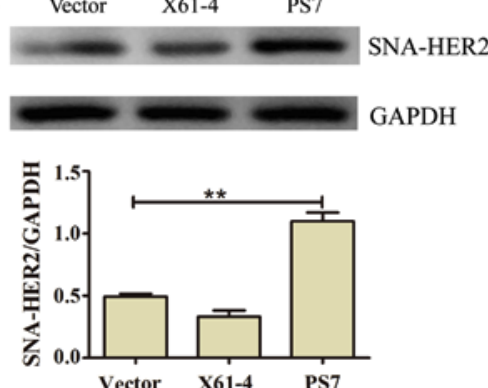

Figure 2. Overexpressing ST6Gal-I impacts the levels of $\alpha 2,6$-linked sialic acids on HER2. (A) The level of $\alpha 2,6$-linked sialic acids was recognized by FITC-conjugated SNA lectin using flow cytometry. (B) The graph depicts the relative fluorescence intensities of the three groups, each of which was analyzed in triplicate ${ }^{* *} \mathrm{P}<0.01$. (C) Cells were stained with FITC-SNA and then assessed for $\alpha 2,6$ sialylation on the cell surface by fluorescence microscopy. Scale bar, $50 \mu \mathrm{m}$. (D) The graph depicts the relative fluorescence intensities of the three groups, each of which was analyzed in triplicate. ${ }^{*} \mathrm{P}<0.05$, ${ }^{* *} \mathrm{P}<0.01$. (E) Sialylated HER2 was harvested and lysed for immunoblotting analysis. ${ }^{* *} \mathrm{P}<0.01$. ST6Gal-I, $\beta$-galactoside $\alpha 2,6$-sialyltransferase; HER2, human epidermal growth factor receptor 2. 

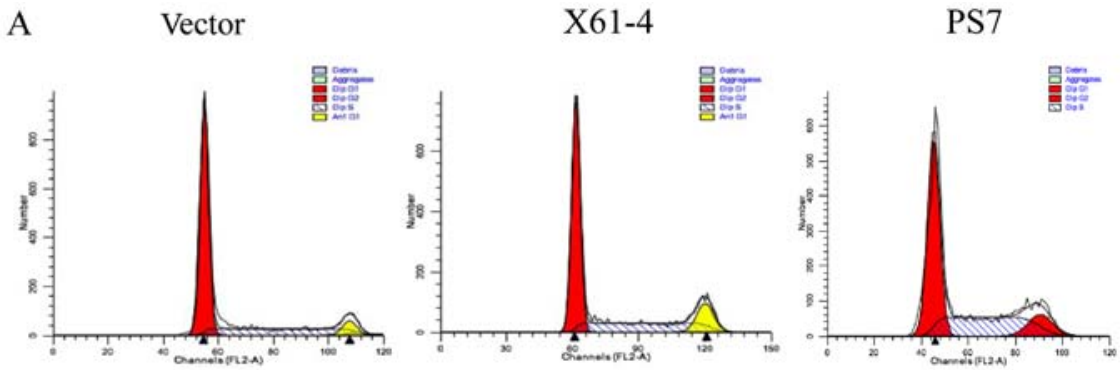

B

$\mathrm{C}$
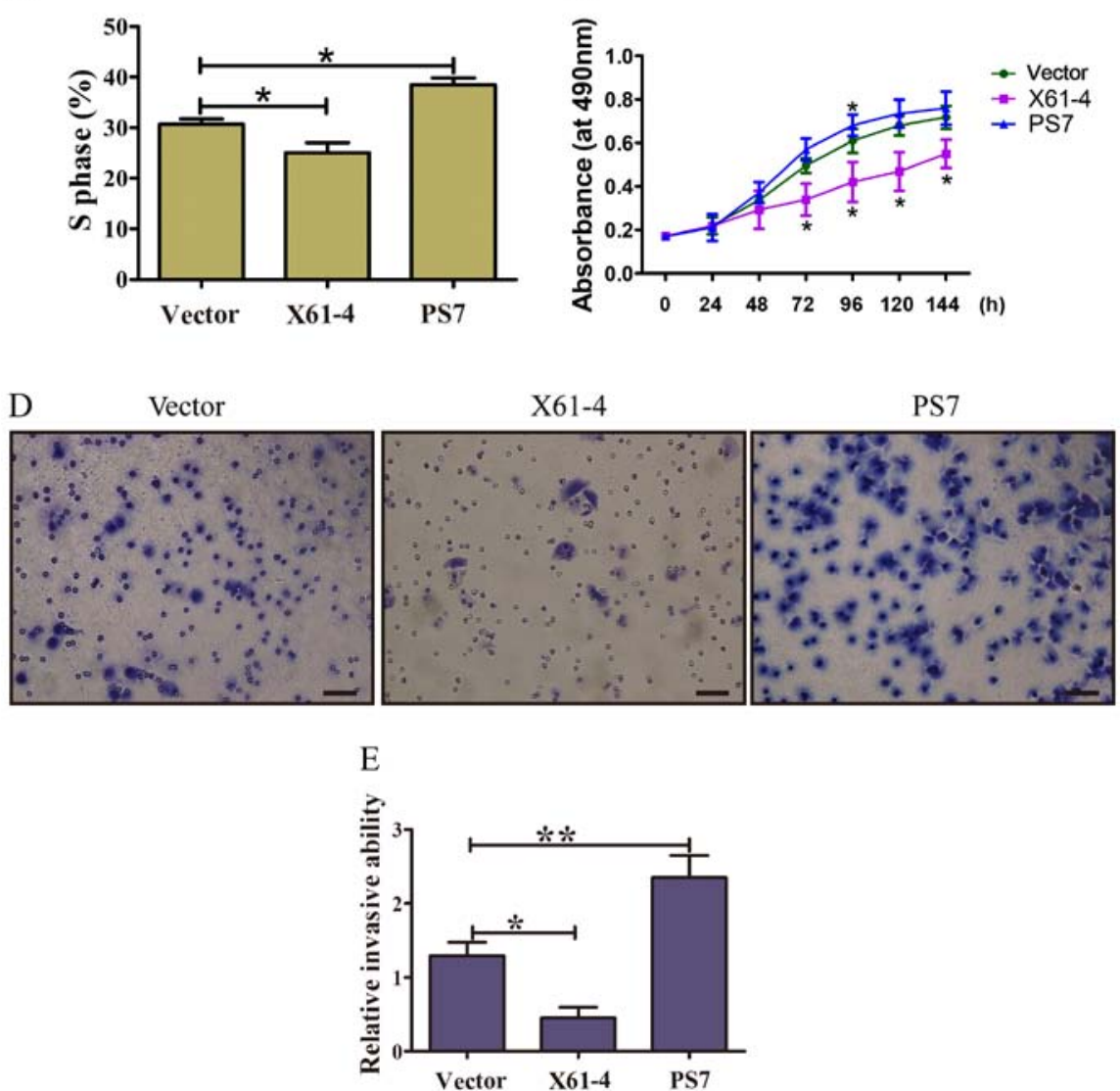

Figure 3. Effect of ST6Gal-I overexpression and ST6Gal-I knockdown on the SGC7901 cell cycle and invasion ability. (A) Representative FACS analysis indicating the cell cycles of SGC7901 cells in which ST6Gal-I was overexpressed or knocked down at $24 \mathrm{~h}$. (B) The graph depicts the quantitative analysis of the cell cycle S phase in SGC7901 cells in which ST6Gal-I was overexpressed or knocked down; each experiment was performed in triplicate. "P<0.05. (C) Cell proliferation in ST6Gal-I-overexpressing or ST6Gal-I knockdown cells under serum deprivation as assessed by CCK-8 assay. (D) Representative invasion assay in SGC7901 cells in which ST6Gal-I was overexpressed or knocked down. Scale bar, $100 \mu \mathrm{m}$. ${ }^{*} \mathrm{P}<0.05$. (E) The graph depicts the relative invasion abilities of the three groups, each of which were analyzed in triplicate. " $\mathrm{P}<0.05,{ }^{* * *} \mathrm{P}<0.01$. ST6Gal-I, $\beta$-galactoside $\alpha 2,6$-sialyltransferase; CCK-8, Cell Counting Kit-8.

GC cells from serum starvation by regulating cell cycle progression, FACS was used to analyze the cell cycles of PS7 cells, X61-4 cells and vector cells. Representative FACS results for the three group cells are shown in Fig. 3A, and FACS data are summarized in Fig. 3B. Among the PS7 cells, $35.07 \%$ were in the $\mathrm{S}$ phase, which was much higher than that in X61-4 cells (26.6\%) and vector control cells (30.71\%). Accordingly, the proportion of PS7 cells remaining in the G2/M phase was $11.66 \%$, compared to $6.74 \%$ for X61-4 cells and $8 \%$ for vector cells $(\mathrm{P}<0.01)$. Serum-starved cells highly expressing ST6Gal-I maintained more cells in the $\mathrm{S}$ phase than low ST6Gal-I-expressing cells, suggesting that ST6Gal-I expression promoted GC cells from the G0/G1 phase into the $\mathrm{S}$ phase, thus enhancing proliferation. Thus, disturbances in ST6Gal-I-mediated sialylation altered cell proliferation.
To further assess whether ST6Gal-I expression promoted the proliferation of GC cells, CCK- 8 assays were conducted. The growth rates of PS7 and X61-4 cloned cells were increased and markedly decreased, respectively, compared to those of vector cells under serum starvation (Fig. 3C). Matrigel-coated Transwell assays were applied to detect the invasion abilities of the cloned cell lines. As shown in Fig. 3D and E, more PS7 clone cells penetrated the Matrigel-coated membrane than vector cells, and fewer ST6Gal-I knockdown cells penetrated the Matrigel-coated membrane than vector cells, indicating that ST6Gal-I-overexpressing cells exhibited a much higher invasion ability than vector cells $(\mathrm{P}<0.05)$. Collectively, these results indicated that ST6Gal-I may be an essential factor promoting GC cell proliferation and invasion. 

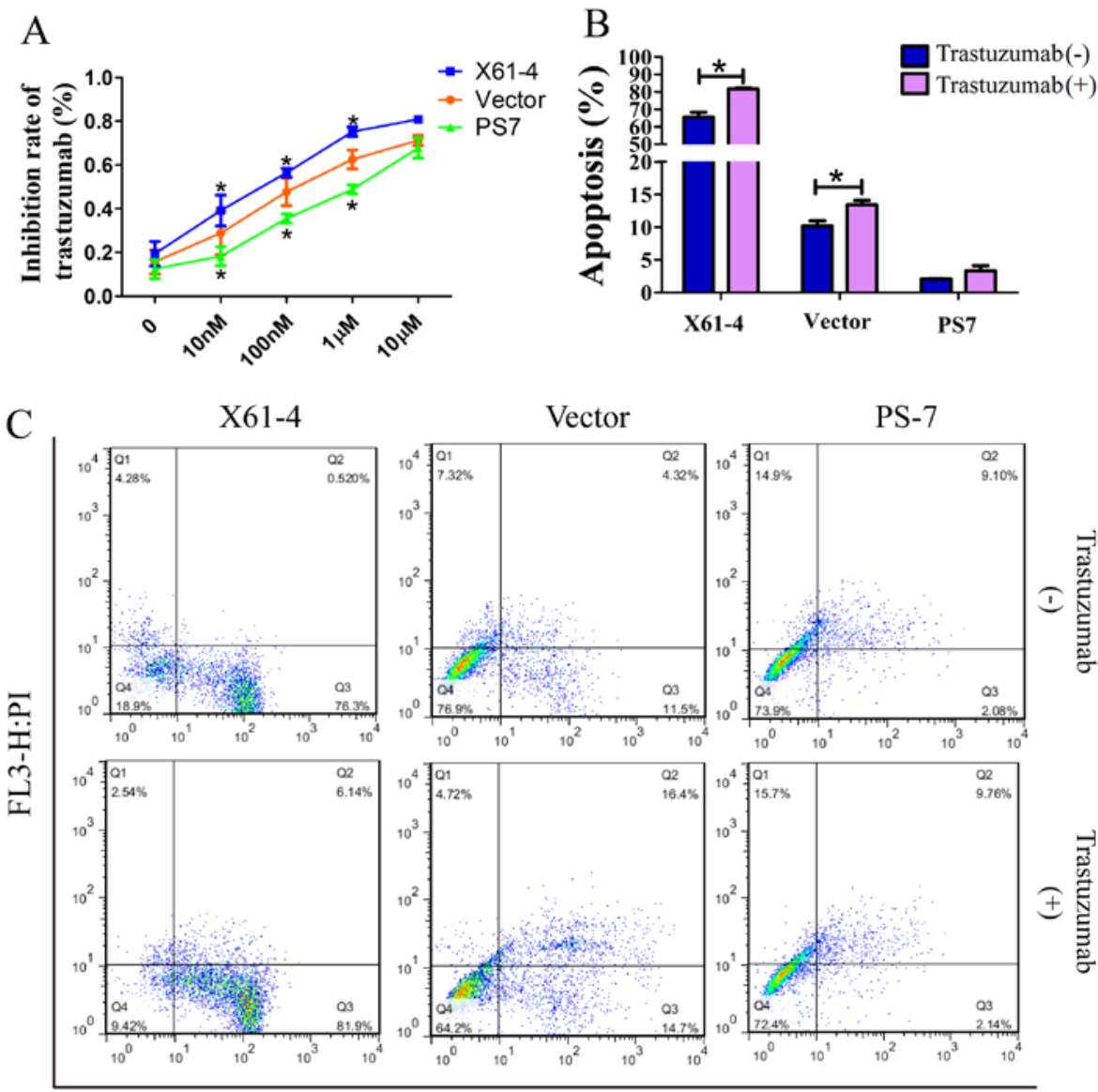

FL1-H:Annexin V-FITC

$\mathrm{D}$
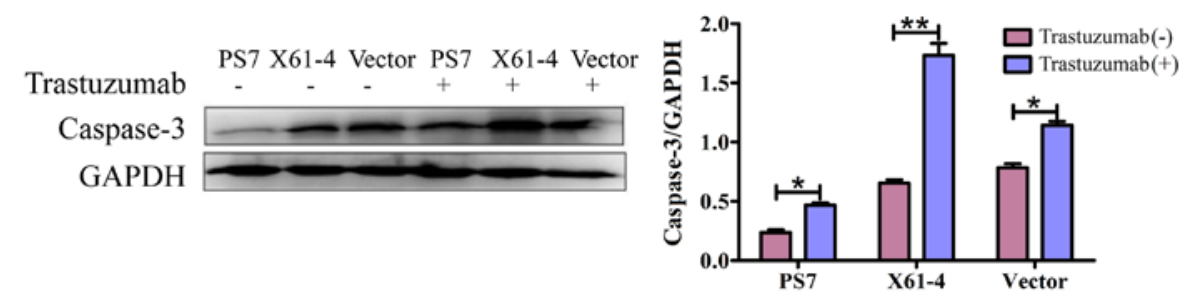

Figure 4. Elevated HER2 $\alpha 2$,6-sialylation increases trastuzumab drug resistance. (A) The trastuzumab inhibition rate in SGC7901 cells in which ST6Gal-I was overexpressed or knocked down was analyzed using a CCK-8 assay. The graph depicts the average of three separate experiments, each of which was performed in triplicate. ${ }^{*} \mathrm{P}<0.05$ vs. the vector. (B) Graph depicting the quantitative analysis of apoptosis populations in SGC7901 cells in which ST6Gal-I was overexpressed or knocked down that were treated with or without trastuzumab; each experiment was performed in triplicate. "P<0.05. (C) Representative apoptosis populations were stained with FITC Annexin V and propidium iodide (PI) in cells in which ST6Gal-I was overexpressed or knocked down that were treated with or without trastuzumab. Lower right quadrant, Annexin V-positive; upper right quadrant, Annexin V-and PI-positive. (D) Caspase-3 levels were detected in cells in which ST6Gal-I was overexpressed or knocked down that were treated with or without trastuzumab by western blotting, and a quantitative analysis of caspase-3 expression is shown, ${ }^{*} \mathrm{P}<0.05,{ }^{* *} \mathrm{P}<0.01$. HER2, human epidermal growth factor receptor 2 ; ST6Gal-I, $\beta$-galactoside $\alpha 2,6$-sialyltransferase; CCK-8, Cell Counting Kit-8.

Elevated HER2 a2,6-sialylation increases trastuzumab drug resistance. For future therapeutic applications, we investigated whether HER2 sialylation decreases apoptosis and enhances resistance to trastuzumab treatment. FACS analysis and a CCK-8 assay were performed to analyze trastuzumab inhibition in GC cells. Trastuzumab inhibition was dose-dependent in each group, and the inhibition effect of trastuzumab on PS7 cells was lower than that on vector cells; however, X61-4 cells were inhibited at a much higher rate than vector cells. When the drug concentration reached $10 \mu \mathrm{M}$, each group exhibited evident cell death. Moreover, FITC-labeled Annexin $\mathrm{V}$ and propidium iodide staining were recorded by FACS to indicate cell apoptosis. As shown in Fig. 4B and C, a small population of PS7 cells were apoptotic (including 2.08\% early-stage apoptosis and $9.10 \%$ late-stage necrosis), whereas a large population of X61-4 cells were apoptotic (76.3\% early-stage apoptosis and $0.52 \%$ late-stage necrosis) under serum starvation. After incubation with trastuzumab, the number of apoptotic PS7 cells was not significantly increased (11.90\% with treatment vs. $11.18 \%$ without), but the number of apoptotic X61-4 cells was distinctly increased $(88.04 \%$ with treatment vs. $76.82 \%$ without). In addition, caspase-3 was reportedly the executioner of apoptosis (28). Our western blot results revealed much higher caspase- 3 expression in X61-4 cells compared to that in PS7 cells, and trastuzumab treatment increased caspase- 3 expression in all three groups compared 


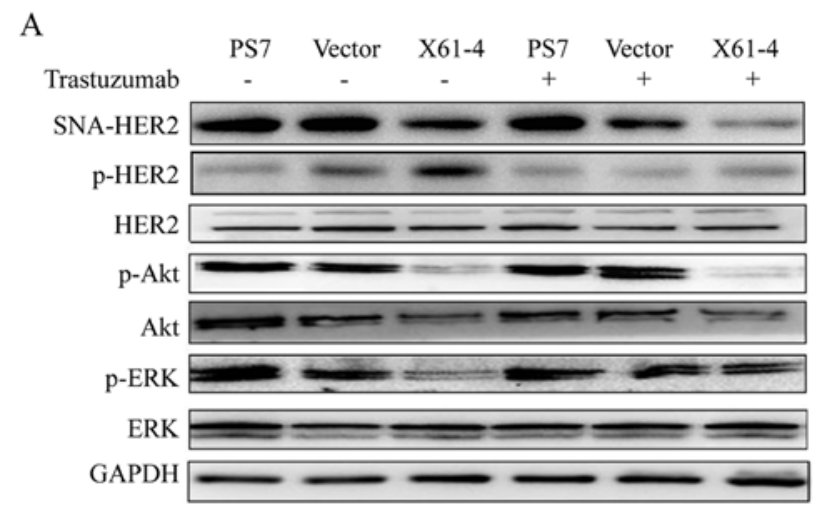

B

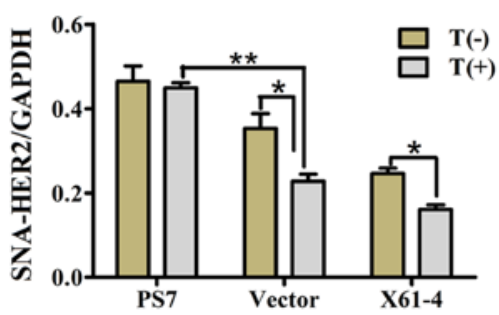

$\mathrm{D}$

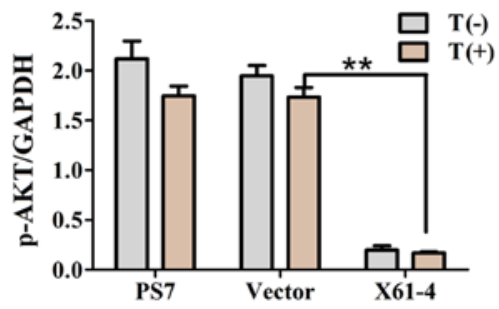

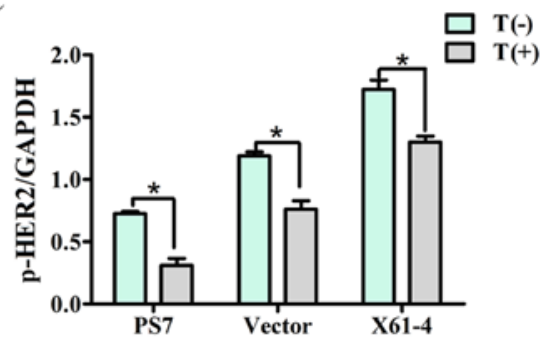

E

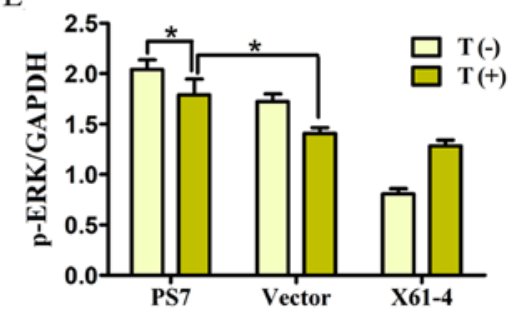

Figure 5. HER $2 \alpha 2,6$-sialylation impacts the Akt and ERK signaling pathways. (A) Cells in which ST6Gal-I was overexpressed or knocked down were treated with or without trastuzumab for $24 \mathrm{~h}$, and the protein levels of SNA-HER2, p-HER2, HER2, p-Akt, Akt, p-ERK, ERK and GAPDH were then measured by western blotting. (B-E) The relative protein intensities of these proteins were detected by ImageJ software. ${ }^{*} \mathrm{P}<0.05,{ }^{* *} \mathrm{P}<0.01$. HER2, human epidermal growth factor receptor 2; ST6Gal-I, $\beta$-galactoside $\alpha 2,6$-sialyltransferase.

to that in cells not treated with trastuzumab (Fig. 4D). Collectively, elevated HER $2 \alpha 2,6$ sialylation reduced cell apoptosis and increased resistance to trastuzumab treatment. In addition, HER $2 \alpha 2,6$ sialylation promoted GC cell survival against many antitumor treatments.

HER2 $\alpha 2,6$ sialylation impacted the Akt and ERK signaling pathways. To investigate how HER2 sialylation affects the downstream signaling pathways exerting drug resistance, we examined the protein levels of HER2, Akt and ERK. HER2 was sialylated by ST6Gal-I in cancer cells, and the SNA-HER2 level in PS7 cells was distinctly higher than that in X61-4 cells. HER2 sialylation was not attenuated with $10 \mu \mathrm{M}$ trastuzumab treatment for $24 \mathrm{~h}$, while HER2 sialylation in X61-4 cells was much lower after trastuzumab treatment (Fig. 5A and B). Phosphorylated HER 2 expression was significantly higher in X61-4 cells than in PS7 cells, and the phosphorylation levels in the three groups were substantially decreased after trastuzumab treatment. The HER 2 protein expression levels in the three groups were similar (Fig. 5A and C). It has been revealed that HER2 activation could initiate the Akt and ERK signaling pathways by increasing Akt and ERK phosphorylation (29). Western blot analyses revealed that the Akt and
ERK phosphorylation levels were significantly higher in PS7 cells than in X61-4 cells and vector cells, and trastuzumab treatment did not alter the phosphorylation levels in any of the groups. Furthermore, the total Akt and Erk repression levels were consistent among all the groups (Fig. 5A, D and E). Collectively, our results indicated that overexpressing ST6Gal-I in cancer cells elevated HER $2 \alpha 2,6$ sialylation, activating the downstream cascade, increasing the phosphorylation of Akt and ERK, and inhibiting the effect of trastuzumab treatment.

\section{Discussion}

HER2 has transmembrane tyrosine kinase activity that promotes cell proliferation and suppresses apoptosis, and HER2 is thereby involved in the pathogenesis and poor outcomes of advanced GCs (5). Overexpressed or aberrant HER2 expression is proposed to promote gastric epithelial cancer cell resistance to HER2-target therapeutics, such as trastuzumab and lapatinib $(30,31)$. In addition, cardiotoxicity is a well-known toxicity caused by high doses of trastuzumab (32). Thus, substantial researchexploring the mechanism of HER2 drug resistance and effective trastuzumab usage in GCs must be performed. 
Evidence has indicated that dysregulated $\alpha 2,6$-sialylation reduces cell apoptosis and promotes drug resistance in cancers $(22,23)$. ST6Gal-I is the key enzyme regulating sialic acid production, and sialic acid is the only sugar in glycoproteins that bears a net negative charge at physiological $\mathrm{pH}$. Due to their terminal location and negative charge, sialic acids can inhibit cell-cell adhesion and enhance adhesion to the extracellular matrix (33). The activity of ST6Gal-I is low or absent in only normal mucosa cells, and its activity is particularly high in metastasizing carcinomas $(17,34)$. We verified that ST6Gal-I was highly expressed in GC, breast cancer and lung cancer cells. ST6Gal-I mRNA and protein expression were assessed after cancer cells were transfected with ST6Gal-I overexpression or ST6Gal-I knockdown plasmids (Fig. 1).

ST6Gal-I adds $\alpha 2,6$-linked sialic acids to select receptors, such as the $\beta 1$ integrin receptor (35), the Fas receptor and TNFR1 death receptors $(20,22)$ and multiple other membrane proteins. In the present study, we made significant progress toward defining the role of ST6Gal-I in GC drug resistance by revealing that HER2 is sialylated by ST6Gal-I (Fig. 2). High HER2 sialylation may impact the cycle of GC cells by enriching the proportion of cells in the $\mathrm{S}$ phase and promoting the invasive ability of cancer cells under serum deprivation (Fig. 3). ST6Gal-I activation has been revealed to promote the expression of cyclin D2 and increase the phosphorylation of $\mathrm{pRb}$, accelerating the transition between the $\mathrm{G} 1$ and $\mathrm{S}$ phases (24). In addition, HER2 overexpression was demonstrated to activate the HER2-PI3K/Akt pathways and induce G2-M arrest in breast cancer $(36,37)$. The results thus far prompted us to further study how sialylated HER2 regulates intracellular cell cycle molecules in GC. Consistent with these results, the caspase-3 expression and incidence of apoptosis in ST6Gal-I-overexpressing cells were low, and the cells exhibited strong resistance to trastuzumab.

The PI3K/Akt pathway provides critical cell mitogenic and survival signals required for tumor progression, and ERK and Akt activation may represent biologically relevant targets for anticancer therapy $(38,39)$. Recent research illustrated that the amplification and/or overexpression of HER2 and phosphorylation of HER2 induced downstream signaling transduction, including activation of the MEK/ERK and PI3K/Akt pathways, promoting trastuzumab resistance in cancer cells $(40,41)$. Results revealed that overexpression of ST6Gal-I induced high HER $2 \alpha 2,6$-sialylation levels and led to low HER2 phosphorylation, while the phosphorylation levels of Akt and ERK were not attenuated. Consequently, ST6Gal-I overexpression increased the proliferation and migration of GC cells. ST6Gal-I overexpression inhibits the phosphorylation of targeted protein sites but may initiate other effector molecules activating the Akt and ERK proteins (42). Thus, multiple mechanisms of resistance may coexist in trastuzumab-resistant cells (43).

In conclusion, our data indicated that amplified or aberrant HER2 expression may not be the only factor underlying trastuzumab resistance in GC. The overexpression level of ST6Gal-I may induce high HER2 $\alpha 2,6$-sialylation, affecting the proliferation, adhesion and invasion of cancer cells and consequently inhibiting cell apoptosis via the PI3K/Akt and ERK pathways. In addition, inhibiting the $\alpha 2,6$-sialylation of HER 2 may be one of numerous therapeutic strategies to overcome or avoid trastuzumab resistance. Candidate strategies in combination with HER2-targeted therapies promoting non-cross resistance and non-overlapping toxicity may be logically ideal.

\section{Acknowledgements}

Not applicable.

\section{Funding}

The present study was supported by the National Natural Science Foundation of China (no. 81071751), the Natural Science Foundation of Guangdong Province (no. 2018A030313639), the Guangzhou Science and Technology Project (no. 201704030059) and the Opening Project of Zhejiang Provincial Top Key Discipline of Pharmaceutical Sciences (no. 2016009).

\section{Availability of data and materials}

The datasets used during the present study are available from the corresponding author upon reasonable request.

\section{Authors' contributions}

SL and XW conceived and designed the study. MZ mainly contributed to the flow cytometry-related experiments, western blot analysis, immunoprecipitation assays and Transwell invasion assay and prepared the first manuscript. YiL, YS and GT mainly contributed to the cell culture. XG, JL and YaL mainly contributed to the RNA isolation and real-time PCR experiments. JW performed the cell transfection. JW and LJ mainly contributed to construct the stable overexpression cell line, JW and $\mathrm{XH}$ mainly contributed to construct the stable knockdown cell line. ZD and XC mainly contributed to the CCK-8 assay. NL provided guidance and solutions for all the experiments and contributed to data statistical analysis. SL, XW and NL reviewed and edited the manuscript. All authors read and approved the manuscript and agree to be accountable for all aspects of the research in ensuring that the accuracy or integrity of any part of the work are appropriately investigated and resolved.

\section{Ethics approval and consent to participate}

Not applicable.

\section{Patient consent for publication}

Not applicable.

\section{Competing interests}

The authors declare that they have no competing interests.

\section{References}

1. Power DG, Kelsen DP and Shah MA: Advanced gastric cancer-slow but steady progress. Cancer Treat Rev 36: 384-392, 2010.

2. Kim HJ, Eun JY, Jeon YW, Yun J, Kim KH, Kim SH, Kim HJ, Lee SC, Bae SB, Kim CK, et al: Efficacy and safety of oxaliplatin, 5-Fluorouracil, and folinic Acid combination chemotherapy as first-line treatment in metastatic or recurrent gastric cancer. Cancer Res Treat 43: 154-159, 2011. 
3. Shah MA, Janjigian YY, Stoller R, Shibata S, Kemeny M, Krishnamurthi S, Su YB, Ocean A, Capanu M, Mehrotra B, et al: Randomized multicenter phase II study of modified docetaxel, cisplatin, and fluorouracil (DCF) versus DCF plus growth factor support in patients with metastatic gastric adenocarcinoma: A study of the US gastric cancer consortium. J Clin Oncol 33 $3874-3879,2015$

4. Jorissen RN, Walker F, Pouliot N, Garrett TP, Ward CW and Burgess AW: Epidermal growth factor receptor: Mechanisms of activation and signalling. Exp Cell Res 284: 31-53, 2003.

5. Boku N: HER2-positive gastric cancer. Gastric Cancer 17: 1-12, 2014.

6. Sui M, Jiao A, Zhai H, Wang Y, Wang Y, Sun D and Li P: Upregulation of miR-125b is associated with poor prognosis and trastuzumab resistance in HER2-positive gastric cancer. Exp Ther Med 14: 657-663, 2017.

7. Gravalos $\mathrm{C}$ and Jimeno A: HER2 in gastric cancer: A new prognostic factor and a novel therapeutic target. Ann Oncol 19 $1523-1529,2008$

8. Tanner M, Hollmén M, Junttila TT, Kapanen AI, Tommola S, Soini Y, Helin H, Salo J, Joensuu H, Sihvo E, et al: Amplification of HER-2 in gastric carcinoma: Association with Topoisomerase II alpha gene amplification, intestinal type, poor prognosis and sensitivity to trastuzumab. Ann Oncol 16: 273-278, 2005.

9. Bang YJ, Van Cutsem E, Feyereislova A, Chung HC, Shen L, Sawaki A, Lordick F, Ohtsu A, Omuro Y, Satoh T, et al; ToGA Trial Investigators: Trastuzumab in combination with chemotherapy versus chemotherapy alone for treatment of HER2-positive advanced gastric or gastro-oesophageal junction cancer (ToGA): A phase 3, open-label, randomised controlled trial. Lancet 376: 687-697, 2010.

10. Taniguchi $\mathrm{N}$ and Korekane $\mathrm{H}$ : Branched N-glycans and their implications for cell adhesion, signaling and clinical applications for cancer biomarkers and in therapeutics. BMB Rep 44: 772-781, 2011.

11. Dall'Olio F, Malagolini N, Trinchera $M$ and Chiricolo $M$ : Mechanisms of cancer-associated glycosylation changes. Front Biosci 17: 670-699, 2012.

12. Schultz MJ, Swindall AF and Bellis SL: Regulation of the metastatic cell phenotype by sialylated glycans. Cancer Metastasis Rev 31: 501-518, 2012

13. Li Y and Chen X: Sialic acid metabolism and sialyltransferases: Natural functions and applications.Appl Microbiol Biotechnol 94: 887-905, 2012.

14. Shaikh FM, Seales EC, Clem WC, Hennessy KM, Zhuo Y and Bellis SL: Tumor cell migration and invasion are regulated by expression of variant integrin glycoforms. Exp Cell Res 314: 2941-2950, 2008.

15. Lu J and Gu J: Significance of $\beta$-galactoside $\alpha 2,6$ sialyltranferase 1 in Cancers. Molecules 20: 7509-7527, 2015.

16. Büll C, Stoel MA, den Brok MH and Adema GJ: Sialic acids sweeten a tumor's life. Cancer Res 74: 3199-3204, 2014.

17. Swindall AF, Londoño-Joshi AI, Schultz MJ, Fineberg N Buchsbaum DJ and Bellis SL: ST6Gal-I protein expression is upregulated in human epithelial tumors and correlates with stem cell markers in normal tissues and colon cancer cell lines. Cancer Res 73: 2368-2378, 2013

18. Dall'Olio F, Chiricolo M, D'Errico A, Gruppioni E, Altimari A, Fiorentino $\mathrm{M}$ and Grigioni WF: Expression of $\beta$-galactoside $\alpha 2,6$ sialyltransferase and of $\alpha 2,6$-sialylated glycoconjugates in normal human liver, hepatocarcinoma, and cirrhosis. Glycobiology 14 $39-49,2004$

19. Lin S, Kemmner W, Grigull S and Schlag PM: Cell surface $\alpha 2,6$ sialylation affects adhesion of breast carcinoma cells. Exp Cell Res 276: 101-110, 2002.

20. Swindall AF and Bellis SL: Sialylation of the Fas death receptor by ST6Gal-I provides protection against Fas-mediated apoptosis in colon carcinoma cells. J Biol Chem 286: 22982-22990, 2011.

21. Zhuo Y, Chammas R and Bellis SL: Sialylation of beta1 integrins blocks cell adhesion to galectin-3 and protects cells against galectin-3-induced apoptosis. J Biol Chem 283: 22177-22185, 2008

22. Liu Z, Swindall AF, Kesterson RA, Schoeb TR, Bullard DC and Bellis SL: ST6Gal-I regulates macrophage apoptosis via a2-6 sialylation of the TNFR1 death receptor. J Biol Chem 286 39654-39662, 2011.

23. Park JJ, Yi JY, Jin YB, Lee YJ, Lee JS, Lee YS, Ko YG and Lee M: Sialylation of epidermal growth factor receptor regulates receptor activity and chemosensitivity to gefitinib in colon cancer cells. Biochem Pharmacol 83: 849-857, 2012.
24. Britain CM, Dorsett KA and Bellis SL: The glycosyltransferase ST6Gal-I protects tumor cells against serum growth factor withdrawal by enhancing survival signaling and proliferative potential. J Biol Chem 292: 4663-4673, 2017.

25. Baek DW, Kang BW, Hwang S, Kim JG, Seo AN, Bae HI, Kwon OK, Lee SS, Chung HY and Yu W: Clinical significance of p53 protein expression, beta-catenin expression and HER2 expression for Epstein-Barr virus-associated gastric cancer. Chonnam Med J 53: 140-146, 2017.

26. Ahmed S, Sami A and Xiang J: HER2-directed therapy: Current treatment options for HER2-positive breast cancer. Breast Cancer 22: 101-116, 2015.

27. Oh DY, Jung K, Song JY, Kim S, Shin S, Kwon YJ, Oh E, Park WY, Song SY and Choi YL: Precision medicine approaches to lung adenocarcinoma with concomitant MET and HER2 amplification. BMC Cancer 17: 535, 2017.

28. Walsh JG, Cullen SP, Sheridan C, Lüthi AU, Gerner C and Martin SJ: Executioner caspase-3 and caspase-7 are functionally distinct proteases.Proc Natl Acad Sci USA 105: 12815-12819, 2008.

29. Joslin EJ, Opresko LK, Wells A, Wiley HS and Lauffenburger DA: EGF-receptor-mediated mammary epithelial cell migration is driven by sustained ERK signaling from autocrine stimulation. J Cell Sci 120: 3688-3699, 2007.

30. Yk W, Cf G, T Y, Z C, Xw Z, Xx L, Nl M and Wz Z: Assessment of $E R B B 2$ and $E G F R$ gene amplification and protein expression in gastric carcinoma by immunohistochemistry and fluorescence in situ hybridization. Mol Cytogenet 4: 14, 2011.

31. Apicella M, Corso S and Giordano S: Targeted therapies for gastric cancer: Failures and hopes from clinical trials. Oncotarget 8: 57654-57669, 2017.

32. Nemeth BT, Varga ZV, Wu WJ and Pacher P: Trastuzumab cardiotoxicity: From clinical trials to experimental studies. Br J Pharmacol 174: 3727-3748, 2016.

33. Yuan Y, Wu L, Shen S, Wu S and Burdick MM: Effect of $\alpha 2,6$ sialylation on integrin-mediated adhesion of breast cancer cells to fibronectin and collagen IV.Life Sci 149: 138-145, 2016.

34. Hedlund M, Ng E, Varki A and Varki NM: $\alpha 2-6$-Linked sialic acids on $\mathrm{N}$-glycans modulate carcinoma differentiation in vivo. Cancer Res 68: 388-394, 2008

35. Hou S, Hang Q, Isaji T, Lu J, Fukuda T and Gu J: Importance of membrane-proximal $N$-glycosylation on integrin $\beta 1$ in its activation and complex formation. FASEB J 30: 4120-4131, 2016.

36. Ma M, Ma Y, Zhang GJ, Liao R, Jiang XF, Yan XX, Bie FJ, Li XB and Lv YH: Eugenol alleviated breast precancerous lesions through HER2/PI3K-AKT pathway-induced cell apoptosis and S-phase arrest.Oncotarget 8: 56296-56310, 2017.

37. Jandial DD, Krill LS, Chen L, Wu C, Ke Y, Xie J, Hoang BH and $\mathrm{Zi} \mathrm{X}$ : Induction of G2M arrest by Flavokawain A, a kava chalcone, increases the responsiveness of HER2-overexpressing breast cancer cells to herceptin. Molecules 22: pii E462, 2017.

38. Bahrami A, Hasanzadeh M, Hassanian SM, ShahidSales S, Ghayour-Mobarhan M, Ferns GA and Avan A: The potential value of the PI3K/Akt/mTOR signaling pathway for assessing prognosis in cervical cancer and as a target for therapy. J Cell Biochem 118: 4163-4169, 2017.

39. Roberts PJ and Der CJ: Targeting the Raf-MEK-ERK mitogen-activated protein kinase cascade for the treatment of cancer. Oncogene 26: 3291-3310, 2007.

40. Teplinsky E and Muggia F: Targeting HER2 in ovarian and uterine cancers: Challenges and future directions. Gynecol Oncol 135: 364-370, 2014.

41. Ikink GJ, Boer M, Bakker ER and Hilkens J: IRS4 induces mammary tumorigenesis and confers resistance to HER2-targeted therapy through constitutive PI3K/AKT-pathway hyperactivation. Nat Commun 7: 13567, 2016.

42. Yen HY, Liu YC, Chen NY, Tsai CF, Wang YT, Chen YJ, Hsu TL, Yang PC and Wong CH: Effect of sialylation on EGFR phosphorylation and resistance to tyrosine kinase inhibition. Proc Natl Acad Sci USA 112: 6955-6960, 2015

43. Köninki K, Barok M, Tanner M, Staff S, Pitkänen J, Hemmilä P, Ilvesaro J and Isola J: Multiple molecular mechanisms underlying trastuzumab and lapatinib resistance in JIMT-1 breast cancer cells. Cancer Lett 294: 211-219, 2010. 